Pacific Journal of Mathematics

HORSESHOE MAPS AND INVERSE LIMITS 


\title{
HORSESHOE MAPS AND INVERSE LIMITS
}

\author{
MARCY BARGE
}

\begin{abstract}
Inverse limits of interval maps are used to show that certain homeomorphisms of the disk into itself factor over a standard $n$-fold horseshoe map on attracting sets and to give a topological classification of the attracting sets of horseshoe maps.
\end{abstract}

1. Introduction. In [S] Smale defined the horseshoe map on the twosphere and gave a complete description of its complicated nonwandering set. Horseshoe maps occur in a wide variety of dynamical systems and an understanding of their properties is crucial in analyzing the dynamics of such systems.

Here we consider the full attracting set for the horseshoe map. This attracting set is a type of snakelike continuum called a Knaster continuum $[\mathbf{B}, \mathbf{W}]$. By realizing these continua as inverse limit spaces for a map of the interval we can, in some situations, construct a particular surjection of one of these continua onto another and, in so doing, provide a dynamical factorization of one horseshoe map over another (at least on attracting sets). This is carried out in $\S \S 2$ and 3. Similar techniques are used by Block in [B1].

In the final section of this paper we use a result of Watkins [W] to give a topological classification of the attracting sets for horseshoe maps.

2. Let $I$ be the interval $I=[0,1]$, and let $D^{2}$ be the two-dimensional disk $D^{2}=I \times I \cup A \cup B$ where $A$ and $B$ are half disks attached to opposite sides $\{0\} \times I$ and $\{1\} \times I$ of the square $I \times I$ as in Figure 1 .

Now let $P: D^{2} \rightarrow I$ by

$$
P(A)=\{0\}, \quad P(B)=\{1\},
$$

and

$$
P((x, y))=x \quad \text { for all }(x, y) \in I \times I \text {. }
$$

For each integer $n \geq 2$ we define an $n$-fold horseshoe map $F_{n}: D^{2} \rightarrow D^{2}$ to be a homeomorphism (into) having the following properties:

(i) $F_{n}\left(P^{-1}(P(z))\right) \subseteq P^{-1}\left(P\left(F_{n}(z)\right)\right)$ for all $z \in D^{2}$; 


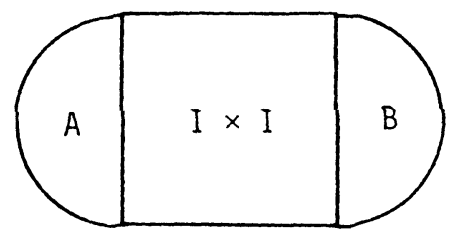

FIGURE 1

(ii) $F_{n}(A) \subseteq$ interior $(A)$ and

$F_{n}(B) \subseteq$ interior $(A)$ for $n$ even,

$F_{n}(B) \subseteq$ interior $(B)$ for $n$ odd;

(iii) for all $x \in I, P^{-1}(x) \cap F_{n}\left(D^{2}\right)$ has exactly $n$ components; and (iv) diameter $\left(F_{n}^{k}\left(P^{-1}(P(z))\right)\right) \rightarrow 0$ uniformly in $z \in D^{2}$ as $k \rightarrow \infty$. The attracting set for the $n$-fold horseshoe map $F_{n}$ is the set $\Lambda_{n}=$ $\bigcap_{k \geq 0} F_{n}^{k}\left(D^{2}\right)$. That is, given $z \in D^{2}$, the distance between $F_{n}^{k}(z)$ and $\Lambda_{n}$ goes to zero as $k$ goes to infinity.

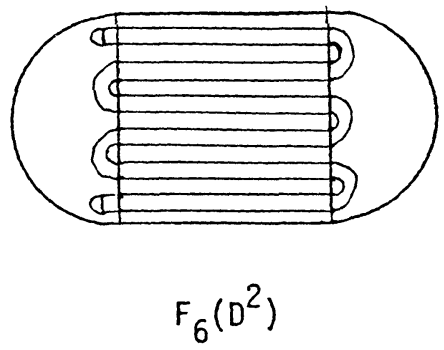

FIGURE 2

For each $n \geq 2, F_{n}$ induces a continuous map of the interval, $f_{n}$ : $I \rightarrow I$, defined by

$$
f_{n}(x)=P\left(F_{n}\left(P^{-1}(x)\right)\right) .
$$

The map $f_{n}$ has the following properties:

$$
\begin{aligned}
& f_{n}(0)=0 ; \\
& f_{n}(1)= \begin{cases}0, & n \text { even }, \\
1, & n \text { odd }\end{cases}
\end{aligned}
$$

and there exists $a_{t} \in I, 0=a_{0}<a_{1}<\cdots<a_{2 n}<a_{2 n+1}=1$ such that $f_{n}$ is strictly monotone on $\left[a_{2 i-1}, a_{2 i}\right]$ for $i=1,2, \ldots, n$ and

$$
f_{n}\left(\left[a_{2 i}, a_{2 i+1}\right]\right)= \begin{cases}\{0\}, & i \text { odd, } \\ \{1\}, & i \text { even } .\end{cases}
$$




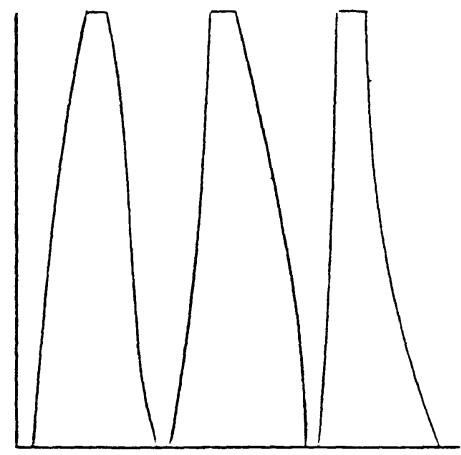

FIGURE 3

Associated with a continuous map of the interval, $f: I \rightarrow I$, is the inverse limit space

$$
(I, f)=\left\{\left(x_{0}, x_{1}, \ldots\right) \mid x_{i} \in I \text { and } f\left(x_{i+1}\right)=x_{i}, i=0,1,2, \ldots\right\} .
$$

We give $(I, f)$ the topology induced by the metric

$$
d\left(\left(x_{0}, x_{1}, \ldots\right),\left(y_{0}, y_{1}, \ldots\right)\right)=\sum_{i=0}^{\infty} \frac{\left|x_{l}-y_{l}\right|}{2^{i}} .
$$

Then $(I, f)$ is a compact connected metric space. Finally, let $\hat{f}:(I, f) \rightarrow$ $(I, f)$ be the homeomorphism defined by

$$
\hat{f}\left(\left(x_{0}, x_{1}, \ldots\right)\right)=\left(f\left(x_{0}\right), x_{0}, x_{1}, \ldots\right) .
$$

Notice that $\hat{f}^{-1}$ is just the shift:

$$
\hat{f}^{-1}\left(\left(x_{0}, x_{1}, x_{2}, \ldots\right)\right)=\left(x_{1}, x_{2}, \ldots\right) .
$$

Now let $F_{n}$ be an $n$-fold horseshoe map of the two disk with attracting set $\Lambda_{n}$ and let $f_{n}$ be the induced map of the interval.

THEOREM 1. The function $\hat{P}: \Lambda_{n} \rightarrow\left(I, f_{n}\right)$ given by

$$
\hat{P}(z)=\left(P(z), P\left(F_{n}^{-1}(z)\right), P\left(F_{n}^{-2}(z)\right), \ldots\right)
$$

is a homeomorphism (onto) and the diagram of homeomorphisms

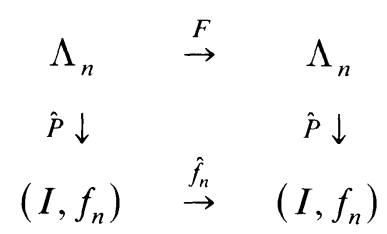

commutes. 
Proof. Since $f_{n}\left(P\left(F_{n}^{-(i+1)}(z)\right)\right)=P\left(F_{n}\left(F_{n}^{-(i+1)}(z)\right)\right)=P\left(F_{n}^{-i}(z)\right)$, we see that $\hat{P}(z)$ is indeed an element of $\left(I, f_{n}\right)$.

$\hat{P}$ is clearly continuous. To see that $\hat{P}$ is one-to-one and onto, let $\mathbf{x}=\left(x_{0}, x_{1}, \ldots\right) \in\left(I, f_{n}\right)$ and let

$$
C_{k}=F_{n}^{k}\left(P^{-1}\left(x_{k}\right)\right) \text { for } k=0,1,2, \ldots
$$

Then $C_{k}$ is a closed, nonempty subset of $D^{2}$ for each $k \geq 0$, and since

$$
F_{n}\left(P^{-1}\left(x_{k+1}\right)\right) \subseteq P^{-1}\left(f_{n}\left(x_{k+1}\right)\right)=P^{-1}\left(x_{k}\right),
$$

we have $C_{k+1} \subseteq C_{k}$ for $k=0,1,2, \ldots$ Thus $\bigcap_{k \geq 0} C_{k}$ is a nonempty set and if $z \in \bigcap_{k \geq 0} C_{k}$, then $P(z)=x_{0}, P\left(F_{n}^{-1}(z)\right)=x_{1}, \ldots$ That is, $\hat{P}(z)=\mathbf{x}$. Moreover, if $\hat{P}(z)=\mathbf{x}$ then $z$ must be in $\bigcap_{k \geq 0} C_{k}$. But since diameter $\left(F_{n}^{k}\left(P^{-1}\left(x_{k}\right)\right)\right) \rightarrow 0$ as $k \rightarrow \infty$ (condition (iv) in the definition of $F_{n}$ ), we have $\bigcap_{k \geq 0} C_{k}=\{z\}$ and $\hat{P}$ is one-to-one as well as onto.

3. Given continuous maps $F: \Lambda \rightarrow \Lambda$ and $G: \Gamma \rightarrow \Gamma$, we say that $F$ factors over $G$ if there is a continuous surjection $H: \Lambda \rightarrow \Gamma$ such that $H \circ F=G \circ H$. If $H$ is a homeomorphism then $F$ and $G$ are said to be conjugate. For example, Theorem 1 states that $\left.F_{n}\right|_{\Lambda_{n}}$ and $\hat{f}_{n}$ are conjugate.

We will see in $\$ 3$ that the attracting sets for any two $n$-fold horseshoe maps $F_{n}$ and $G_{n}$ are homeomorphic. It is not necessarily the case, however, that $F_{n}$ and $G_{n}$ are conjugate on their attracting sets. Nonetheless, there is a "standard" $n$-fold horseshoe map $S_{n}$ such that every other $n$-fold horseshoe map factors over $S_{n}$ (on attracting sets). Actually every $(n+2 k)$-fold horseshoe map $F_{n+2 k}$ factors over $S_{n}$ on attracting sets and, in fact, maps that in rough detail resemble an $n$-fold horseshoe map factor over $S_{n}$ on attracting sets.

In order to be more specific we will work on the level of interval maps. Let $s_{n}$ be a continuous map of the interval $I=[0,1]$ with the following properties: there are points $b_{i}, 0=b_{0}<b_{1}<\cdots<b_{2 n}<$ $b_{2 n+1}=1$ such that

$$
s_{n}\left(\left[b_{2 i}, b_{2 i+1}\right]\right)= \begin{cases}\{0\}, & i \text { odd } \\ \{1\}, & i \text { even }\end{cases}
$$

and $s_{n}$ is linear on $\left[b_{2 i-1}, b_{2 i}\right], i=1,2, \ldots$ Now let $t_{n}$ be any continuous map of the interval satisfying: there exists points $a_{i}, 0=a_{0}<a_{1}<\cdots$ $<a_{2 n}<a_{2 n+1}=1$ such that

$$
t_{n}\left(\left[a_{2 i}, a_{2 i+1}\right]\right)=\left\{\begin{array}{ll}
\{0\}, & i \text { odd, } \\
\{1\}, & i \text { even, }
\end{array} \quad i=0, \cdots, 2 n .\right.
$$

Notice that $t_{n}\left(\left[a_{2 i-1}, a_{2 i}\right]\right)=[0,1]$ for $i=1, \ldots, n$ : but we make no assumptions about how $t_{n}$ behaves on $\left[a_{2 i-1}, a_{2 i}\right]$. 


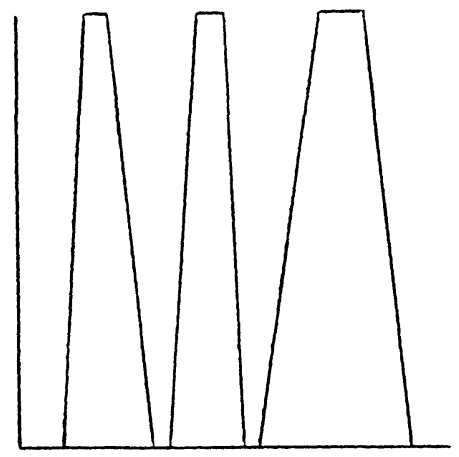

Figure 4

Graph of $s_{6}$

THEOREM 2. Given $s_{n}$ and $t_{n}$ as above, there is a continuous surjection $h: I \rightarrow I$ such that $s_{n} \circ h=h \circ t_{n}$ on $I$.

Proof. So that we have room for future subscripting, let $s=s_{n}$ and $t=t_{n}$.

We will construct the surjection $h$ in steps. Assume that $n \geq 2$ is even. For odd $n$ the construction is much the same.

Step 1. Let $\mathscr{D}_{0}=\bigcup_{i=0}^{n}\left[a_{2 i}, a_{2 i+1}\right]$. In this step we simply define $h$ on $\mathscr{D}_{0}$ such that $\left.h\right|_{\left[a_{2 i}, a_{2 i+1}\right]}$ is an increasing homeomorphism from $\left[a_{2 i}, a_{2 i+1}\right]$ onto $\left[b_{2 i}, b_{2 i+1}\right]$ for each $i=0,1, \ldots, n$. Denote $h \mid \mathscr{D}_{0}$ by $h_{0}$. Then it is clear that for $x \in \mathscr{D}_{0}, s\left(h_{0}(x)\right)=h_{0}(t(x))$.

Step 2. Let $\mathscr{D}_{m}=t^{-m}\left(\mathscr{D}_{0}\right)$ for $m=0,1,2, \ldots$ and let $\mathscr{D}=\bigcup_{m \geq 0} \mathscr{D}_{m}$. Since $t\left(\mathscr{D}_{0}\right) \subseteq \mathscr{D}_{0}$ we have $\mathscr{D}_{m+1} \supseteq \mathscr{D}_{m}$ for all $m \geq 0$. In this step we extend $h_{0}$ to $\mathscr{D}$. To do this first define

$$
s_{i}^{-1}: I \rightarrow\left[b_{2 i-1}, b_{2 i}\right], \quad i=1, \ldots, n
$$

to be the inverse of $\left.s\right|_{\left[b_{2 i-1}, b_{21}\right]}$. Also, for $x \in I$ such that $t^{k}(x) \in I-\mathscr{D}_{0}$ for $k=0,1, \ldots, m-1$, define

$$
\alpha_{k}=\alpha_{k}(x)=i \text { provided } t^{k-1}(x) \in\left(a_{2 i-1}, a_{i}\right) \text { for } k=1, \ldots, m \text {. }
$$

Now let $x \in \mathscr{D}$. Then $x \in \mathscr{D}_{0}$ or $x \in \mathscr{D}_{m}-\mathscr{D}_{m-1}$ for some $m \geq 1$. If $x \in \mathscr{D}_{0}$ let $h(x)=h_{0}(x)$. If $x \in \mathscr{D}_{m}-\mathscr{D}_{m-1}$ for some $m \geq 1$, let

$$
h(x)=s_{\alpha_{1}}^{-1} \circ \cdots \circ s_{\alpha_{m}}^{-1} \circ h_{0} \circ t^{m}(x)
$$

where $\alpha_{k}=\alpha_{k}(x), k=1, \ldots, m$. This is well defined since $x \notin \mathscr{D}_{m-1}$. We will show that $h$ is continuous on $\mathscr{D}$ and that $s \circ h=h \circ t$ on $\mathscr{D}$. 
If $x \in \mathscr{D}_{m+k}-\mathscr{D}_{m+k-1}$, then $t^{k}(x) \in \mathscr{D}_{m}-\mathscr{D}_{m-1}$ and

$$
\begin{aligned}
h(x) & =s_{\alpha_{1}}^{-1} \circ \cdots \circ s_{\alpha_{k}}^{-1} \circ s_{\alpha_{k+1}}^{-1} \circ \cdots \circ s_{\alpha_{m+k}}^{-1} \circ h_{0} \circ t^{m} \circ t^{k}(x) \\
& =s_{\alpha_{1}}^{-1} \circ \cdots \circ s_{d \alpha_{k}}^{-1} \circ\left(s_{\alpha_{k+1}}^{-1} \circ \cdots \circ s_{\alpha_{m+k}}^{-1} \circ h_{0} \circ t^{m}\right) \circ t^{k}(x) \\
& =s_{\alpha_{1}}^{-1} \circ \cdots \circ s_{\alpha_{k}}^{-1} \circ h \circ t^{k}(x)
\end{aligned}
$$

since $\alpha_{l}\left(t^{k}(x)\right)=\alpha_{i+k}(x)$ for $i=1, \ldots, m$. In particular, for $x \in \mathscr{D}_{m+1}$ $-\mathscr{D}_{m}$ and $m \geq 0$, we have $h(x)=s_{\alpha_{1}}^{-1} \circ h \circ t(x)$ so that $s \circ h(x)=$ $s \circ s_{\alpha_{1}}^{-1} \circ h \circ t(x)=h \circ t(x)$. Thus $s \circ h=h \circ t$ on all of $\mathscr{D}$.

Let $x \in \mathscr{D}$. Then $t^{m}(x)=0$ for some $m \geq 0$ ( $n$ even). Thus, if $y$ is sufficiently close to $x, t^{m}(y) \in\left[0, a_{1}\right) \subseteq \mathscr{D}_{0}$. It follows that $\mathscr{D}$ is open.

To establish the continuity of $h$ on $\mathscr{D}$, we will first show that $h$ is continuous on $\mathscr{D}_{1}$. If $x \in \mathscr{D}_{1}-\mathscr{D}_{0}$ then for $y$ sufficiently close to $x$ (and $\left.y \in \mathscr{D}_{1}\right)$ we have $y \in \mathscr{D}_{1}-\mathscr{D}_{0}, \alpha_{1}=\alpha_{1}(y)=\alpha_{1}(x)$, and

$$
h(y)=s_{\alpha_{1}}^{-1} \circ h_{0} \circ t(y) \simeq s_{\alpha_{1}}^{-1} \circ h_{0} \circ t(x)=h(x) .
$$

If $x \in \operatorname{interior}\left(\mathscr{D}_{0}\right)$ then for $y$ sufficiently close to $x, y \in \operatorname{interior}\left(\mathscr{D}_{0}\right)$, and

$$
h(y)=h_{0}(y) \simeq h_{0}(x)=h(x) .
$$

If $x \in \mathscr{D}_{0}-\operatorname{interior}\left(\mathscr{D}_{0}\right)=\left\{a_{1}, a_{2}, \ldots, a_{2 n}\right\}$, say $x=a_{J}$, then $h(x)=$ $h_{0}(x)=b_{i}$. Now, note that if $j$ is odd then $s_{j}^{-1}(0)=b_{2 j-1}$ and $s_{j}^{-1}(1)=$ $b_{2 j}$, and if $j$ is even then $s_{j}^{-1}(0)=b_{2 j}$ and $s_{j}^{-1}(1)=b_{2 j-1}$. Let $y \in \mathscr{D}_{1}-$ $\mathscr{D}_{0}$ be close to $x$. We consider four cases.

If $i \equiv 0 \bmod 4$ then $y \in\left(a_{i-1}, a_{l}\right), \alpha_{1}=i / 2$ is even, $t\left(a_{l}\right)=0$ so $t(y) \simeq 0$, and

$$
h(y)=s_{\alpha_{1}}^{-1} \circ h_{0} \circ t(y) \circ s_{\alpha_{1}}^{-1} \circ h_{0}(0) \simeq s_{\alpha_{1}}^{-1}(0)=b_{2 \alpha_{1}}=b_{i}=h(x) .
$$

If $i \equiv 1 \bmod 4$ then $y \in\left(a_{i}, a_{i+1}\right), \alpha_{1}=(i-1) / 2+1$ is odd, $t\left(a_{i}\right)$ $=0$ so $t(y) \simeq 0$, and

$$
h(y)=s_{\alpha_{1}}^{-1} \circ h_{0} \circ t(y) \simeq s_{\alpha_{1}}^{-1} \circ h_{0}(0) \simeq s_{\alpha_{1}}^{-1}(0)=b_{2 \alpha_{1}-1}=b_{\imath}=h(x) .
$$

If $i \equiv 2 \bmod 4$ then $y \in\left(a_{i-1}, a_{i}\right), \alpha_{1}=i / 2$ is odd, $t\left(a_{i}\right)=1$ so $t(y) \simeq 1$, and

$$
h(y)=s_{\alpha_{1}}^{-1} \circ h_{0} \circ t(y) \simeq s_{\alpha_{1}}^{-1} \circ h_{0}(1) \simeq s_{\alpha_{1}}^{-1}(1)=b_{2 \alpha_{1}}=b_{i}=h(x) .
$$

If $i \equiv 3 \bmod 4$ then $y \in\left(a_{l}, a_{i+1}\right), \alpha_{1}=(i-1) / 2+1$ is even, $t\left(a_{i}\right)$ $=1$ so $t(y) \simeq 1$, and

$$
h(y)=s_{\alpha_{1}}^{-1} \circ h_{0} \circ z(y) \simeq s_{\alpha_{1}}^{-1} \circ h_{0}(1) \simeq s_{\alpha_{1}}^{-1}(1)=b_{2 \alpha_{1}-1}=b_{l}=h(x) .
$$

Thus we see that $h$ is continuous on $\mathscr{D}_{1}$. 
Now let $x \in \mathscr{D}_{m}-\mathscr{D}_{m-1}$ for some $m \geq 1$. Then $t^{m+1}(x) \in$ interior $\left(\mathscr{D}_{0}\right)$ so if $y$ is sufficiently close to $x$ then $t^{m+1}(y) \in \mathscr{D}_{0}$ and $y \in$ $\mathscr{D}_{m+1}$. Also, for $y$ close to $x, t^{k}(y) \in I-\mathscr{D}_{0}$ for $k=0,1, \ldots, m-1$ and $\alpha_{k}(y)=\alpha_{k}(x)=\alpha_{k}$ for $k=1, \ldots, m$. Then

$$
\begin{aligned}
h(y) & =\left.s_{\alpha_{1}}^{-1} \circ \cdots \circ s_{\alpha_{m}}^{-1} \circ h\right|_{\mathscr{D}_{1}} \circ t^{m}(y) \\
& \left.\simeq s_{\alpha_{1}}^{-1} \circ \cdots \circ s_{\alpha_{m}}^{-1} \circ h\right|_{\mathscr{D}_{1}} \circ t^{m}(x)=h(x)
\end{aligned}
$$

since $h$ is continuous on $\mathscr{D}_{1}$. Thus, $h$ is continuous on $\mathscr{D}$.

Step 3. We now extend $h$ to $\overline{\mathscr{D}}$.

Let $x \in \overline{\mathscr{D}}-\mathscr{D}$. Since $x \notin \mathscr{D}, t^{k}(x) \in I-\mathscr{D}_{0}$ for all $k \geq 0$. Thus $\alpha_{k}(x)$ is defined for all $k \geq 1$ and for each $m \geq 0$ there is an $\varepsilon_{m}>0$ such that $\varepsilon_{m} \rightarrow 0$ as $m \rightarrow \infty$ and, if $|x-y|<\varepsilon_{m}$, then $\alpha_{k}(y)$ is defined and equals $\alpha_{k}(x)$ for $k=1,2, \ldots, m$.

Now for $y, z \in \mathscr{D}$ such that $|x-y|<\varepsilon_{m}$ and $|x-z|<\varepsilon_{m}$ we have $\alpha_{k}(y)=\alpha_{k}(z)=\alpha_{k}$ for $k=1, \ldots, m$ and

$$
\begin{aligned}
& h(y)=s_{\alpha_{1}}^{-1} \circ \cdots \circ s_{\alpha_{m}}^{-1} \circ h \circ t^{m}(y), \\
& h(z)=s_{\alpha_{1}}^{-1} \circ \cdots \circ s_{\alpha_{m}}^{-1} \circ h \circ t^{m}(z) .
\end{aligned}
$$

Let $\lambda_{i}$ be the slope of $\left.s\right|_{\left[a_{2 l-1}, a_{2 i}\right]}$ and let $\lambda=\min _{i=1, \ldots, n}\left(\left|\lambda_{i}\right|\right)$. Then $\lambda>1$ and

$$
\operatorname{diameter}\left(s_{\alpha_{1}}^{-1} \circ \cdots \circ s_{\alpha_{m}}^{-1}(1)\right) \leq \frac{1}{\lambda^{m}} .
$$

Hence, letting $B_{\varepsilon}(x)=\{y \in I|| x-y \mid<\varepsilon\}$, we see that

$$
\text { diameter }\left(h\left(B_{\varepsilon_{m}}(x) \cap \mathscr{D}\right)\right) \rightarrow 0 \quad \text { as } m \rightarrow \infty \text {. }
$$

Thus $h$ extends continuously to $\overline{\mathscr{D}}$. We see in fact that

$$
h(x)=\lim _{m \rightarrow \infty} s_{\alpha_{1}}^{-1} \circ \cdots \circ s_{\alpha_{m}}^{-1}(z) \text { for every } z \in I .
$$

Clearly $s \circ h=h \circ t$ on $\overline{\mathscr{D}}$.

Step 4. We now extend $h$ to all of $I$.

Let $x \in I-\overline{\mathscr{D}}$ and let $U$ be the component of $I-\overline{\mathscr{D}}$ containing $x$. Say $U=(y, z)$. Since $\mathscr{D}$ is itself open, $y$ and $z$ are in $\bar{D}-\mathscr{D}$. Now, it must be the case that $\alpha_{k}(y)=\alpha_{k}(z)$ for all $k \geq 1$ (these numbers are defined since $y, z \notin \mathscr{D})$; otherwise there would be a $k \geq 0$ such that $t^{k}([y, z]) \supseteq\left[a_{2 i}, a_{2 i+1}\right]$ for some $i$ in which case $U \cap \mathscr{D}_{k} \neq 0$. But $U \subseteq$ $I-\overline{\mathscr{D}} \subseteq I-\mathscr{D}_{k}$. 
Thus we see that

$$
\begin{aligned}
h(y) & =\lim _{m \rightarrow \infty}\left(s_{\alpha_{1}(y)}^{-1} \circ \cdots \circ s_{\alpha_{m}(y)}^{-1}(w)\right) \\
& =\lim _{m \rightarrow \infty}\left(s_{\alpha_{1}(z)}^{-1} \circ \cdots \circ s_{\alpha_{m}(z)}^{-1}(w)\right)=h(z)
\end{aligned}
$$

for any $w \in I$ (see Step 3). Now define

$$
h(x)=\lim _{m \rightarrow \infty}\left(s_{\alpha_{1}(x)}^{-1} \circ \cdots \circ s_{\alpha_{m}(x)}^{-1}(x)\right)
$$

for $x \in I-\overline{\mathscr{D}}$. Then $h$ is continuous on all of $I$. It is a simple matter to check that $s \circ h=h \circ t$ on all of $I$.

Corollary 3. Suppose that $s_{n}$ and $t_{n}$ are as in Theorem 2 except that, in addition, $t_{n}$ is linear on $\left[a_{2 i-1}, a_{2 i}\right]$ for $i=1,2, \ldots, n$. Then there is $a$ homeomorphism $h: I \rightarrow I$ such that $s_{n} \circ h=h \circ t_{n}$ on all of $I$. That is, $s_{n}$ and $t_{n}$ are conjugate on $I$.

Proof. The surjection $h$ constructed in Theorem 2 is a homeomorphism in this case. In fact, if one constructs a continuous surjection $k$ : $I \rightarrow I$ as in the theorem, beginning with $k_{0}: \bigcup_{i=0}^{n}\left[b_{2 i}, b_{2 i+1}\right] \rightarrow$ $\bigcup_{i=0}^{n}\left[a_{2 i}, a_{2 i+1}\right]$ given by $k_{0}=h_{0}^{-1}$ and reversing the roles of $s$ and $t$, then $k=h^{-1}$.

Now suppose that $S_{n}: D^{2} \rightarrow D^{2}$ in an $n$-fold horseshoe map with the property that the induced map on the interval, $s_{n}: I \rightarrow I$, is linear on $\left[b_{2 i-1}, b_{2 i}\right], \quad i=1,2, \ldots, n$ (as in the earlier definition of $s_{n}$ ). Let $T: D^{2} \rightarrow D^{2}$ be any homeomorphism satisfying: there exists a projection $\Phi: D^{2} \rightarrow I$ such that $T\left(\Phi^{-1}(\Phi(z))\right) \subseteq \Phi^{-1}(\Phi(T(z)))$ for all $z \in D^{2}$; diameter $\left(T^{k}\left(\Phi^{-1}(\Phi(z))\right)\right) \rightarrow 0$ uniformly in $z \in D^{2}$ as $k \rightarrow \infty$; and the induced map of the interval $t: I \rightarrow I$ has the property that

$$
t\left(\left[a_{2 i}, a_{2 i+1}\right]\right)= \begin{cases}\{0\} & \text { for } i \text { even } \\ \{1\} & \text { for } i \text { odd }\end{cases}
$$

for some $0=a_{0}<a_{1}<\cdots<a_{2 n+1}=1$.

Let $\Lambda_{n}=\bigcap_{k \geq 0} S_{n}^{k}\left(D^{2}\right)$ and $\Gamma=\bigcap_{k \geq 0} T^{k}\left(D^{2}\right)$.

COROllaRy 4. There is a continuous surjection $H: \Gamma \rightarrow \Lambda_{n}$ such that the diagram

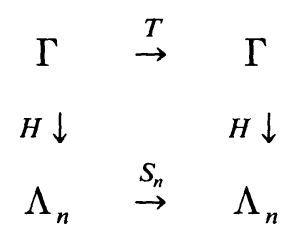

commutes. That is, T on $\Gamma$ factors over $S_{n}$ on $\Lambda_{n}$. 
Proof. Theorems 1 and 2 provide the following commutative diagram

$\begin{array}{ccc}\Gamma & \stackrel{T}{\rightarrow} & \Gamma \\ \hat{\Phi} \downarrow & & \hat{\Phi} \downarrow \\ (I, t) & \stackrel{\hat{t}}{\rightarrow} & (I, t) \\ \hat{h} \downarrow & & \hat{h} \downarrow \\ \left(I, s_{n}\right) & \stackrel{\hat{s}_{n}}{\rightarrow} & \left(I, s_{n}\right) \\ \hat{P} \uparrow & & \hat{P} \uparrow \\ \Lambda_{n} & \stackrel{S_{n}}{\rightarrow} & \Lambda_{n}\end{array}$

$\hat{\Phi}$ and $\hat{P}$ are homeomorphisms and $\hat{h}:(I, t) \rightarrow\left(I, s_{n}\right)$ is the continuous surjection given by

$$
\hat{h}\left(\left(x_{0}, x_{1}, \ldots\right)\right)=\left(h\left(x_{0}\right), h\left(x_{1}\right), \ldots\right)
$$

where $h$ is given by Theorem 2. Let $H=\hat{P}^{-1} \circ \hat{h} \circ \hat{\Phi}$.

Thus we see that if $m \geq n$ and $m$ and $n$ have the same parity, then the $m$-fold horseshoe map $F_{m}$ factors over the standard $n$-fold horseshoe map $S_{n}$ on their attracting sets.

In the next section we give a topological classification of the spaces $\Lambda_{n}$.

4. We first define a standard model for the spaces $\Lambda_{n}$.

Let $g_{n}: I \rightarrow I, n \geq 2$, be defined by

$$
g_{n}\left(\frac{i}{n}\right)=\left\{\begin{array}{ll}
0, & i \text { even, } \\
1, & i \text { odd },
\end{array} \quad i=0, \ldots, n,\right.
$$

and $g_{n}$ is linear on $[i / n,(i+1) / n], i=0, \ldots, n-1$. Let

$$
\left(I, g_{n}\right)=\left\{\left(x_{0}, x_{1}, \ldots\right) \mid x_{i} \in I, g_{n}\left(x_{i+1}\right)=x_{i}, i=0,1, \ldots\right\}
$$

be the inverse limit space. Another description of the space $\left(I, g_{n}\right)$ is as follows. Let $\Sigma_{n}=\left\{\left(\alpha_{1}, \alpha_{2}, \ldots\right) \mid \alpha_{1} \in\{1, \ldots, n\}\right\}$ and give $\Sigma_{n}$ the topology induced by the metric

$$
d\left(\left(\alpha_{1}, \alpha_{2}, \ldots\right),\left(\beta_{1}, \beta_{2}, \ldots\right)\right)=\sum_{i=1}^{\infty} \frac{\left|\alpha_{\imath}-\beta_{t}\right|}{2^{i}} .
$$

Let $I \times \Sigma_{n}$ have the product topology and define $P_{n}: I \times \Sigma_{n} \rightarrow\left(I, g_{n}\right)$ by $P_{n}(x, \boldsymbol{\alpha})=\mathbf{x}$ where $\mathbf{x}=\left(x_{0}, x_{1}, \ldots\right)$ is the unique element of $\left(I, g_{n}\right)$ such that $x_{0}=x$ and $x_{i} \in\left[\alpha_{i} / n,\left(\alpha_{i}+1\right) / n\right]$. 
$P_{n}$ is at most two-to-one and if we define the equivalence relation by $(x, \boldsymbol{\alpha}) \sim(y, \boldsymbol{\beta})$ if and only if $P_{n}((x, \boldsymbol{\alpha}))=P_{n}((y, \boldsymbol{\beta}))$ then $\left(I \times \Sigma_{n}\right) / \sim$ with the quotient topology is homeomorphic with $\left(I, g_{n}\right)$.

More specifically, in $I \times \Sigma_{n}$ we identify $(x, \boldsymbol{\alpha})$ with $(y, \boldsymbol{\beta})$ if and only if one of the following applies.

(1) If $\alpha_{1}=2 j-1$ and $\beta_{1}=2 j$ for some $j$ and $\alpha_{i}=\beta_{l}$ for $i>1$ then $(1, \boldsymbol{\alpha}) \sim(1, \boldsymbol{\beta})$.

(2) If $n$ is odd and $\alpha_{i}=\beta_{i}=n$ for $i=1, \ldots, m ; \alpha_{m+1}=2 j-1$, $\beta_{m+1}=2 j$ for some $j$; and $\alpha_{i}=\beta_{i}$ for $i>m+1$; then $(1, \alpha) \sim(1, \boldsymbol{\beta})$.

(3) If $\alpha_{t}=\beta_{l}=1$ or $i=1,2, \ldots, m ; \alpha_{m+1}=2 j$ and $\beta_{m+1}=2 j$ for some $j$; and $\alpha_{i}=\beta_{i}$ for $i>m+1$; then $(0, \boldsymbol{\alpha}) \sim(0, \boldsymbol{\beta})$.

(4) If $n$ is even and $\alpha_{i}=\beta_{i}=1$ for $i=1, \ldots, m ; \alpha_{m+1}=\beta_{m+1}=n$; $\alpha_{m+2}=2 j-1$ and $\beta_{m+2}=2 j$ for some $j$; and $\alpha_{l}=\beta_{i}$ for $i>m+2$; then $(0, \boldsymbol{\alpha}) \sim(0, \boldsymbol{\beta})$.

Now let $f_{n}$ be the interval map corresponding to an $n$-fold horseshoe $\operatorname{map} F_{n}$.

THEOREM 5. $\left(I, f_{n}\right)$ is homeomorphic with $\left(I, g_{n}\right)$.

Proof. Let $J=\left[\frac{1}{4}, \frac{3}{4}\right] \subseteq I$ and let $\Sigma_{n}$ be as above. Define $Q_{n}$ : $J \times \Sigma_{n} \rightarrow\left(I, f_{n}\right)$ by $Q_{n}(x, \boldsymbol{\alpha})=\mathbf{x}$ where $\mathbf{x}=\left(x_{0}, x_{1}, \ldots\right)$ is the unique element of $\left(I, f_{n}\right)$ such that $x_{0}=2\left(x-\frac{1}{4}\right)$ and $x_{i} \in\left[a_{2 \alpha_{t}-1}, a_{2 \alpha_{t}}\right]$. Here $0=a_{0}<a_{1}<\cdots<a_{2 n+1}=1$ as in the description of $f_{n}$ in $\S 1$. Then $Q_{n}$ is a homeomorphism of $J \times \Sigma_{n}$ onto its image. $\left(I, f_{n}\right)-Q_{n}\left(J \times \Sigma_{n}\right)$ is a collection of arcs. We extend $Q_{n}$ to $\left(I \times \Sigma_{n}\right) / \sim$ by mapping the arcs in $\left(\left(I \times \Sigma_{n}\right) / \sim\right)-\left(J \times \Sigma_{n}\right)$ onto the corresponding $\operatorname{arcs}$ in $\left(I, f_{n}\right)-$ $Q_{n}\left(J \times \Sigma_{n}\right)$.

For example, let $\boldsymbol{\alpha}$ and $\boldsymbol{\beta}$ be as in Case (1) above in the description of the identifications made in $\left(I \times \Sigma_{n}\right) / \sim$. Then $Q_{n}\left(\left(\frac{3}{4}, \boldsymbol{\alpha}\right)\right)=$ $\left(1, a_{4 j-2}, \ldots\right)=\mathbf{x}$ and $Q_{n}\left(\left(\frac{3}{4}, \boldsymbol{\beta}\right)\right)=\left(1, a_{4 j-1}, \ldots\right)=\mathbf{y}$ for some $j$. Then

$$
\begin{aligned}
A_{\mathbf{\alpha}}=\left\{\mathbf{t}=\left(1, t_{1}, t_{2}, \ldots\right) \in\left(I, f_{n}\right) \mid a_{4 J-2} \leq t_{1}\right. & \leq a_{4 j-1}, \\
t_{l} & \left.\in\left[a_{2 \alpha_{\iota}-1}, a_{2 \alpha_{l}}\right]\right\}
\end{aligned}
$$

is an arc joining $\mathbf{x}$ to $\mathbf{y}$ in $\left(I, f_{n}\right)$. Now let $Q_{n}$ map the arc

$$
\left(\left[\frac{3}{4}, 1\right] \times\{\boldsymbol{\alpha}\} \cup\left[\frac{3}{4}, 1\right] \times\{\boldsymbol{\beta}\}\right) / \sim
$$

in $\left(I \times \Sigma_{n}\right) / \sim$ onto $A_{\alpha}$ by

$$
Q_{n}((s, \boldsymbol{\alpha}))=\mathbf{t}=\left(1, t_{1}, t_{2}, \ldots\right) \in A_{\boldsymbol{\alpha}}
$$


where

$$
t_{1}=\left(a_{4 j-1}-a_{4 j-2}\right)\left(2 s-\frac{3}{2}\right)+a_{4 j-2}
$$

and

$$
Q_{n}((s, \boldsymbol{\beta}))=\mathbf{t}=\left(1, t_{1}, t_{2}, \ldots\right) \in A_{\alpha}
$$

where

$$
t_{1}=\left(a_{4 j-2}-a_{4 j-1}\right)\left(2 s-\frac{3}{2}\right)+a_{4 j-1} .
$$

If we extend $Q_{n}$ over all the arcs in $\left(\left(I \times \Sigma_{n}\right) / \sim\right)-\left(J \times \Sigma_{n}\right)$ in this way (depending on Case 1, 2, 3 or 4), the result is a homeomorphism of $\left(I \times \Sigma_{n}\right) / \sim$ onto $\left(I, f_{n}\right)$.

The spaces $\left(I, g_{n}\right)$ are classified in the following theorem of Watkins [W].

THEOREM. $\left(I, g_{n}\right)$ is homeomorphic wiht $\left(I, g_{m}\right)$ if and only if $n$ and $m$ have exactly the same prime factors.

COROLlaRy. $\Lambda_{n}$ is homeomorphic with $\Lambda_{m}$ if and only if $n$ and $m$ have exactly the same prime factors.

\section{REFERENCES}

[B] R. H. Bing, Snake-like continua, Duke Math. J., (3) 18 (1951), 653-663.

[B1] L. Block, Diffeomorphisms obtained from endomorphisms, Trans. Amer. Math. Soc., 214 (1975), 403-413.

[S] S. Smale, Diffeomorphisms With Many Periodic Points, in Differential and Combinatorial Topology - A Symposium in Honor of Marston Morse, (Stewart S. Cairns, Ed.). Princeton, New Jersey: Princeton University Press, 1965, pp. 63-80.

[W] W. T. Watkins, Homeomorphic classification of certain inverse limit spaces with open bonding maps, Pacific J. Math., (2) 103 (1982), 589-601.

Received May 10, 1984 and in revised form December 12, 1984.

UNIVERSITY OF WYOMING

LARAMIE, WY 82071 



\section{PACIFIC JOURNAL OF MATHEMATICS EDITORS}

V. S. VARADARAJAN (Managing Editor)
University of California
Los Angeles, CA 90024
HEBERT CLEMENS
University of Utah
Salt Lake City, UT 84112
CHARLES R. DEPRIMA
California Institute of Technology
Pasadena, CA 91125

\section{R. FINN}

Stanford University

Stanford, CA 94305

HeRmanN FlaschKa

University of Arizona

Tucson, AZ 85721

RAMESH A. GANGOLli

University of Washington

Seattle, WA 98195

ROBION KIRBY

University of California

Berkeley, CA 94720

\section{C. MoOre}

University of California

Berkeley, CA 94720

H. SAMELSON

Stanford University

Stanford, CA 94305

HAROLD STARK

University of California, San Diego

La Jolla, CA 92093

ASSOCIATE EDITORS
R. ARENS
E. F. BECKENBACH
B. H. NeUmanN
F. WOLF
K. Yoshida (1906-1982)

\section{SUPPORTING INSTITUTIONS}

UNIVERSITY OF ARIZONA

UNIVERSITY OF BRITISH COLUMBIA

CALIFORNIA INSTITUTE OF TECHNOLOGY

UNIVERSITY OF CALIFORNIA

MONTANA STATE UNIVERSITY

UNIVERSITY OF NEVADA, RENO

NEW MEXICO STATE UNIVERSITY

OREGON STATE UNIVERSITY
UNIVERSITY OF OREGON

UNIVERSITY OF SOUTHERN CALIFORNIA

STANFORD UNIVERSITY

UNIVERSITY OF HAWAII

UNIVERSITY OF TOKYO

UNIVERSITY OF UTAH

WASHINGTON STATE UNIVERSITY

UNIVERSITY OF WASHINGTON 


\section{Pacific Journal of Mathematics}

\section{Vol. 121, No. $1 \quad$ November, 1986}

Om P. Agrawal, Douglas Napier Clark and Ronald George Douglas,

Invariant subspaces in the polydisk $\ldots \ldots \ldots \ldots \ldots \ldots \ldots \ldots \ldots \ldots$

Christoph Bandt and Gebreselassie Baraki, Metrically invariant measures

on locally homogeneous spaces and hyperspaces $\ldots \ldots \ldots \ldots \ldots \ldots \ldots$

Marcy Mason Barge, Horseshoe maps and inverse limits ..............29

Russell Gene Bilyeu, Robert Richard Kallman and Paul Weldon Lewis,

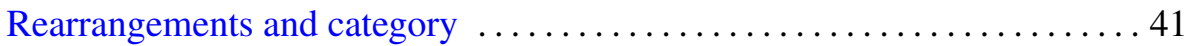

Jean Bourgain, A problem of Douglas and Rudin on factorization . . .......47

Hernan Cendra, A normal form and integration in finite terms for a class of

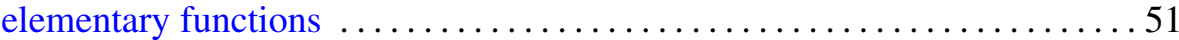

Ky Fan, The angular derivative of an operator-valued analytic function . . . . 67

Gerhard Gierz, On the Dunford-Pettis property of function modules of

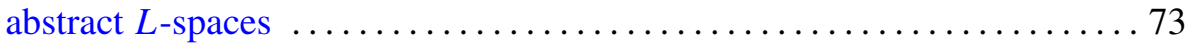

Gabriel Katz, On polynomial generators in the algebra of complex functions

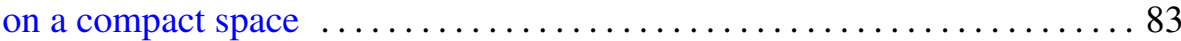

Ridgley Lange, Duality and asymptotic spectral decompositions $\ldots \ldots \ldots . .93$

Anthony To-Ming Lau and Peter F. Mah, Quasinormal structures for certain spaces of operators on a Hilbert space ................... 109

R. Daniel Mauldin, Correction: "The set of continuous nowhere differentiable functions"

Alan Harvey Mekler and Saharon Shelah, $\omega$-elongations and Crawley's problem

Alan Harvey Mekler and Saharon Shelah, The solution to Crawley's problem

Richard Rochberg, Deformation of uniform algebras on Riemann surfaces

Joseph Roitberg, On weak epimorphisms in homotopy theory

Jesús M. Ruiz, A remark on fields with the dense orbits property

Henry Wente, Counterexample to a conjecture of H. Hopf

David G. Wright, Rigid sets in $E^{n}$ 